\title{
Narrativa
}

\section{La ricezione della letteratura italiana contemporanea in Germania e il concetto di inter- e transculturalità}

\section{Susanne Kleinert}

\section{(2) OpenEdition}

\section{Journals}

\section{Edizione digitale}

URL: https://journals.openedition.org/narrativa/873

DOI: $10.4000 /$ narrativa.873

ISSN: 2804-1224

\section{Editore}

Presses universitaires de Paris Nanterre

\section{Edizione cartacea}

Data di pubblicazione: 1 décembre 2016

Paginazione: 163-172

ISBN: 978-2-84016-266-7

ISSN: $1166-3243$

\section{Notizia bibliografica digitale}

Susanne Kleinert, «La ricezione della letteratura italiana contemporanea in Germania e il concetto di inter- e transculturalità», Narrativa [Online], 38 | 2016, online dal 01 janvier 2022, consultato il 22 février 2022. URL: http://journals.openedition.org/narrativa/873 ; DOI: https://doi.org/10.4000/narrativa.873

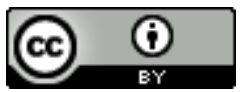

Narrativa est mise à disposition selon les termes de la Licence Creative Commons Attribution 4.0 International. 


\section{La ricezione della letteratura italiana contemporanea in Germania e il concetto di inter- e transculturalità}

\section{LE TRADUZIONI DELLA LETTERATURA ITALIANA CONTEMPORANEA IN GERMANIA}

La ricezione della letteratura italiana gode di una lunga tradizione in Germania. Molti classici del Novecento sono stati recepiti velocemente, come testimoniano le traduzioni di Pirandello, Svevo, Calvino, Pasolini e molti altri autori reperibili sul catalogo della Biblioteca Nazionale (Deutsche Nationalbibliothek) ${ }^{1}$. Anche autori difficili da tradurre come Gadda e Stefano D'Arrigo sono stati tradotti in tedesco, ma con un certo ritardo ${ }^{2}$. Il mercato tedesco è di un notevole interesse economico per le case editrici italiane perché costituisce una possibilità d'accesso anche al pubblico austriaco e svizzero, oltre a quello della Germania. D'altro canto, la fiera del libro di Francoforte, che ogni anno dedica una sezione speciale a un paese straniero, ha mostrato un'attenzione particolare per il mercato librario italiano, invitando l'Italia a presentare la sua letteratura in quanto ospite onorario già nel 1988, un anno prima della Francia.

Per quanto riguarda la ricezione della letteratura italiana uscita dopo il 2000 - il periodo su cui si concentrano gli ultimi numeri della rivista Narrativa - si può constatare facilmente che vengono tradotte le opere degli autori che godono di una popolarità più o meno notevole sul mercato internazionale,

1. Il catalogo online contiene i dati della Deutsche Nationalbibliothek dal 1913 in poi; cfr. www.dnb.de/DE/Kataloge/kataloge_node.html . Sulla ricezione della letteratura italiana del Novecento in Germania, cfr. KLESCZEWSKI, Reinhard, KöNIG, Bernhard (a cura di), Italienische Literatur in deutscher Sprache: Bilanz und Perspektiven, Tübingen, Narr Verlag, 1990.

2. Le traduzioni di Quer pasticciaccio brutto de Via Merulana (Milano, Garzanti, 1957) e della Cognizione del dolore (Torino, Einaudi, 1963) di Gadda sono uscite rispettivamente nel 1961 e nel 1964 presso la casa editrice Piper di Monaco di Baviera; per Horcynus Orca di Stefano d'Arrigo (Milano, Mondadori, 1975), bisognerà aspettare il 2015 (Francoforte sul Meno, edizioni Fischer). 
come Carmine Abate, Alessandro Baricco, Stefano Benni, Andrea Camilleri ${ }^{3}$, Gianni Celati, Erri De Luca, Umberto Eco, Claudio Magris, Dacia Maraini, Antonio Tabucchi e Sandro Veronesi. Di solito, i libri di questi autori sono tradotti velocemente ${ }^{4}$.

Non possiamo pretendere in questa sede che la nostra ricerca bibliografica sia esaustiva; malgrado queste riserve, si può osservare che una grande quantità di titoli italiani tradotti in Germania sono dei gialli. In alcuni casi l'appartenenza a questo genere è sottolineata dal titolo tedesco. Una strategia commerciale che punta a un pubblico di potenziali turisti è facilmente reperibile in un sottotitolo quale "ein Toscana-Krimi", cioè "un giallo ambientato in Toscana". Il genere del giallo regionalistico, cioè ambientato in un contesto regionale, è molto diffuso in Germania e non stupisce il fatto che le case editrici cerchino di inserire le traduzioni in questo genere. Il turismo e la possibilità di collegare un titolo tradotto a un marchio già conosciuto appaiono quindi come due fattori in generale favorevoli alla decisione di tradurre o meno un testo italiano. Bisogna aggiungere che vengono anche tradotti dei gialli con una forte impronta critica come quelli di Giancarlo De Cataldo o di Massimo Carlotto.

Un caso interessante mi pare essere quello della ricezione della letteratura sarda che è recepita indipendentemente dall'appartenenza dei vari titoli al genere del giallo. Sono stati tradotti in tedesco molti scrittori sardi, come Milena Agus, Marcello Fois, Michela Murgia, Salvatore Niffoi, Giorgio Todde e Flavio Soriga, e questo confermerebbe la tendenza alla traduzione "turistica", ossia quella di chi voglia approfondire l'incontro con una cultura diversa leggendo dei romanzi ambientati in una regione meta di vacanze come la Sardegna.

Tradurre delle opere di autori giovani o poco conosciuti è sempre un rischio per una casa editrice. Perciò è interessante osservare che esistono delle traduzioni di autori che affrontano temi come la crisi economica e il precariato: si pensi a Cordiali saluti (2005) di Andrea Bajani, Generažione Mille Euro (2006) di Alessandro Rimassa e Antonio Incorvaia o anche Il mondo deve sapere (2006) di Michela Murgia.

Sul mercato del libro in Germania sono presenti inoltre vari romanzi di Niccolò Ammaniti, Paola Capriolo, Ascanio Celestini, Elena Ferrante, Giorgio Fontana, Giuseppe Genna, Paolo Giordano, Fleur Jaeggy, Davide Longo, Maurizio Maggiani, Paolo Maurensig, Margaret Mazzantini, Melania Mazzucco,

3. Di Camilleri sono stati tradotti sia i gialli che i romanzi storici a partire dal 1998.

4. I dati bibliografici sono reperibili sia su Amazon.de che sull'Opac della Deutsche Nationalbibliothek. 
Francesca Melandri, Valeria Parrella, Antonio Pennacchi, Alessandro Piperno, Tiziano Scarpa, Antonio Scurati, Giorgio Vasta, Mariolina Venezia, Serena Vitale, oltre a vari romanzi del duo Monaldi e Sorti e tre titoli di Wu Ming. ̇̀ stata pubblicata anche un'antologia di racconti di giovani autori a cura di Paola Gallo e Dalia Oggero, due editori della narrativa italiana di Einaudi ${ }^{6}$. Dopo il successo della pubblicazione in lingua tedesca di Gomorra di Roberto Saviano, uscita nel 2009, sono stati tradotti anche altri testi dell'autore. Le opere di Walter Siti, che cominciano a essere studiate dalla critica accademica tedesca, non esistono ancora in versione tedesca. Lo stesso vale per i romanzi di Mauro Covacich, del quale si conta solo la traduzione del suo libro su Trieste (Trieste sottosopra, 2006).

Per quanto riguarda la poesia, sono state pubblicate alcune traduzioni presso la prestigiosa casa editrice Carl Hanser, fra cui opere di Patrizia Cavalli, Anna Maria Carpi, Milo De Angelis e di Valerio Magrelli. Inoltre è da menzionare l'antologia Die Erschließung des Lichts, uscita nel 2013 presso la stessa casa editrice Hanser?.

La letteratura cosiddetta migrante non è stata tradotta ad eccezione di pochi casi. La casa editrice Wagenbach, conosciuta per la traduzione di autori come Pier Paolo Pasolini e Luigi Malerba, ha tradotto due romanzi di Amara Lakhous. Altre case editrici hanno pubblicato traduzioni di Ornela Vorpsi, Anilda Ibrahimi e Fabio Geda ${ }^{8}$. Inoltre, nel 2008 è uscita la traduzione tedesca della raccolta di poesie Maldiluna di Gezim Hajdari.

5. Per i tipi della casa editrice Assoziation A (Amburgo e Berlino), politicamente orientata a sinistra, sono uscite, tra il 2015 e il 2016, le traduzioni di $Q$ (Torino, Einaudi, 1999) di Luther Blissett, di 54 (Torino, Einaudi, 2002) e di Altai (Torino, Einaudi, 2009) di Wu Ming, oltre a ristampe di precedenti traduzioni di testi di Nanni Balestrini. Per la ricezione di Wu Ming si può quindi constatare un certo ritardo. Le traduzioni di alcune opere di Valerio Evangelisti sono uscite in case editrici note per le loro politiche commerciali come Goldmann. Inoltre sono stati tradotti molti volumi di genere Fantasy dell'autrice Licia Troisi presso la casa editrice Heyne che punta a una letteratura di massa.

6. Nel 2011 la casa editrice Wagenbach di Berlino ha pubblicato $A$ casa nostra, un'antologia che contiene testi di Paolo Giordano, Nicola Lagioia, Andrea Bajani, Peppe Fiore, Antonella Lattanzi, Gabriele Pedullà, Paolo Cognetti, Valeria Parrella e Silvia Avallone, autori che ormai sono parzialmente conosciuti anche in Germania.

7. Quest'antologia contiene poesie di poeti canonizzati e ben conosciuti in Germania come Ungaretti e Montale, ma anche di scrittori meno noti come Vittorio Sereni, Franco Fortini, Mario Luzi, Giovanni Raboni, Valerio Magrelli, di autori del "Gruppo 63", nonché di Antonella Anedda e Patrizia Cavalli.

8. Di Lakhous sono stati tradotti Scontro di civiltà per un ascensore a Piazza Vittorio (Roma, e/o, 2006) e Divorzio all'islamica in Viale Marconi (Roma, e/o, 2010), di Vorpsi 
A lungo termine, le traduzioni e le case editrici hanno un ruolo preponderante nel processo di diffusione e ricezione di una letteratura straniera, ma non va trascurata l'importanza di altre istituzioni che meriterebbero un'analisi a parte. In tale senso, vanno segnalati soprattutto gli Istituti italiani di cultura che invitano molti autori contemporanei e li fanno conoscere al pubblico tedesco. Inoltre bisognerebbe studiare i programmi di vari festival come l'Internationales Literaturfestival di Berlino o gli incontri organizzati dalla Fiera del Libro di Francoforte o da vari atenei. Tra questi sono da notare incontri con autori non ancora pubblicati in Germania, come per esempio Igiaba Scego e Cristina Ali Farah?

\section{LA RICEZIONE ACCADEMICA DELLA LETTERATURA ITALIANA CONTEMPORANEA}

La struttura degli studi italianistici in Germania si distingue da quella di altri Paesi ed è dovuta alla tradizione degli studi delle lingue e letterature romanze in Germania. Qui infatti, non esistono dipartimenti specifici d'italianistica, salvo a Monaco di Baviera. Gli studi italianistici, ispanistici e "gallo-romanzi", cioè di lingua e letteratura francese, formano un insieme di filologie chiamato "Romanistik". In genere, uno studioso d'italianistica è al contempo un esperto di linguistica o letteratura francese. Normalmente, il grado di specializzazione di un italianista tedesco è inferiore rispetto a quello dei suoi omologhi francesi o italiani. Di solito, un dipartimento di romanistica dispone di un'unica cattedra di letteratura italiana che include italiano e francese. Infine, in Germania esiste una tradizione abbastanza forte di studi di letteratura italiana medievale e rinascimentale, ma non iper-contemporanea. Tutti questi fattori non sono favorevoli a una ricezione approfondita della letteratura attuale in ambito accademico. Malgrado questi inconvenienti che rendono difficile una ricezione aggiornata, esistono diversi studi sulla letteratura italiana più recente. Senza la pretesa di farne un elenco esaustivo, limitiamoci a richiamare qui alcuni esempi tratti dalla bibliografia annuale Romanische Bibliographie, dai convegni organizzati dall'Associazione degli Italianisti Tedeschi DIV (Deutscher Italianistenverband) e dalle riviste

Il paese dove non si muore mai (Torino, Einaudi, 2005) e La mano che non mordi (Torino, Einaudi, 2007), di Ibrahimi Rosso come una sposa (Torino, Einaudi, 2008) e di Fabio Geda Nel mare ci sono i coccodrilli (Milano, Baldini, 2010).

9. Ali Farah e Scego sono state invitate all'Università di Würzburg nel 2012 e nel 2011. Scego ha partecipato al Festival internazionale di letteratura di Berlino nel 2010 e alla Fiera del Libro di Francoforte nel 2015 ed è anche invitata dagli Istituti italiani di cultura di Berlino e Francoforte. 
Romanistisches Jabrbuch, Italienisch e Zibaldone: Zeitschrift für italienische Kultur der Gegenwart $^{10}$.

Esistono studi sugli autori più noti a livello internazionale quali Umberto Eco, Dacia Maraini e Antonio Tabucchi. Vanno ricordati almeno il libro di Thomas Stauder, che contiene le sue interviste con Umberto Eco ${ }^{11}$, gli studi di Barbara Kuhn su Tabucchi ${ }^{12}$ e il libro della studiosa austriaca Birgit Wagner sulla letteratura e cultura sarda uscito nel $2008^{13}$. Il catalogo della Deutsche Nationalbibliothek elenca poi una grande quantità di studi su Italo Calvino. Tra le tesi di dottorato di ricerca sulla letteratura italiana ultra-contemporanea figurano gli studi di Viktoria Adam su Cavazzoni e di Stefanie Rubenis sul tema della violenza sul corpo femminile in varie opere di Laura Pugno, Alda Teodorani e Isabella Santacroce ${ }^{14}$; l'analisi di Christiane Ebner su Veronesi uscita nel 2013 e il libro di Claudia Jacobi sulla ricezione di Proust in Serge Doubrovsky, Carmen Martín Gaite e Walter Siti, uscito nel 2016 ${ }^{15}$. Vanno ricordati inoltre due saggi su Wu Ming e, nell'ambito del convegno recente degli italianisti tedeschi, un contributo su Mauro Covacich ${ }^{16}$. Un'attenzione particolare è stata dedicata all'argomento del "ritorno alla realtà" in un convegno organizzato da Michael

10. La rivista Romanistisches Jabrbuch contiene un elenco annuale delle tesi di dottorato di ricerca e delle tesi di abilitazione alla libera docenza e fornisce così un panorama degli studi svolti nei dipartimenti tedeschi.

11. StAuder, Thomas, Gespräche mit Umberto Eco aus drei Jahrzebnten, Berlin, LIT, 2012.

12. Kunn, Barbara, "Schreiben im Zwischen-Raum. Tristano-Tabucchis 'Buch der Unruhe"', in Behrens, Rudolf, Stillers, Rainer (a cura di), Inquietudini: Gestalt, Funktion und Darstellung eines affektiven Musters in der italienischen Literatur, Heidelberg, Winter, 2010, pp. 247-268. I riferimenti bibliografici di altri studi di Barbara Kuhn su Tabucchi sono reperibili sulla sua pagina personale nel sito dell'Università Cattolica di Eichstätt-Ingolstadt dove insegna.

13. Wagner, Birgit, Sardinien. Insel im Dialog. Texte, Diskurse, Filme, Tübingen, Francke, 2008.

14. ADAm, Viktoria, Der lunatische Roman und die Poetik der Ambivalen:: narratologische Untersucbungen zum Romanwerk. Ermanno Cavazzonis, Heidelberg, Winter, 2014; RuBENIS, Stefanie, Gewalt am Franenkörper in der scrittura femminile nera: Zum Werk von Laura Pugno, Alda Teodorani und Isabella Santacroce, Frankfurt a.M., Lang, 2012.

15. EBNer, Christiane, Amore per la letteratura - Passione per il cinema: Eine medienkomparatistische Studie zu Sandro Veronesis Romanwerk, Berlin, LIT, 2012; JACOBI, Claudia, Proust dixit? Réceptions de La Recherche dans P autofiction de Serge Doubrovsky, Carmen Martín Gaite et Walter Siti, Göttingen, V\&R unipress, 2016.

16. Cfr. Tiller, Elisabeth, "'Non c'è niente di nuovo sotto il sole'? Politische Unruhe und Literatur zur Jahrtausendwende", in BeHRens, Stillers (a cura di), Inquietudini, cit., pp. 269-293; SCHAEFER, Christina, "Wu Ming, New Italian Epic und der Entwurf eines neuen Italien", in FöCKING, Marc, SchwArZE, Michael (a cura di), Una gente di lingua, di memorie e di cor. Italienische Literatur und schwierige nationale Einheit von Macbiavelli bis Wu 
Schwarze, Christian Rivoletti e Jobst Welge, tenutosi a Costanza nel 2014, con vari contributi sulla letteratura italiana ultra-contemporanea ${ }^{17}$. La rivista Italienisch pubblica regolarmente interviste ad autori italiani in lingua italiana e, meno regolarmente, anche saggi su autori ultra-contemporanei: in questo senso, segnaliamo un breve contributo di Vikoria Adam su Nicolai Lilin e un saggio di Christine Ott su Elena Ferrante ${ }^{18}$. La rivista Zibaldone dedica inoltre vari numeri a temi specifici come le regioni d'Italia e pubblica articoli in genere piuttosto brevi su testi letterari attinenti a queste tematiche.

\section{IL CONCETTO DI INTER- E TRANSCULTURALITÀ IN GERMANIA E LA RICEZIONE ACCADEMICA DELLA LETTERATURA DELLA MIGRAZIONE}

Nel panorama generale della ricezione accademica della letteratura italiana ultra-contemporanea, un elemento attira l'attenzione: la ricezione, negli ultimi anni, della letteratura migrante e transculturale. Questo nuovo fenomeno riguarda soprattutto la ricerca. Se nel 2002 Maria Cristina Mauceri constatava l'assenza della letteratura della migrazione nei curricula universitari in Germania e Francia ${ }^{19}$, la situazione nel frattempo forse non è cambiata di molto per quanto riguarda l'insegnamento degli italianisti tedeschi che in genere si attengono allo studio di autori canonici. Tuttavia, se guardiamo con attenzione le attività di ricerca attualmente in corso, e se guardiamo i programmi di insegnamento sulla letteratura postcoloniale, transculturale e/o di migrazione, svolti in università come Amburgo, Augsburg, Berlino, Mannheim, Saarbrücken e Würzburg, possiamo immaginare che in futuro la letteratura della migrazione sarà più presente non solo nei programmi didattici, ma si svilupperà maggiormente anche come ambito di ricerca.

A differenza degli studi sulle letterature francofone che esistono in Germania già da molti anni, la ricezione della letteratura di autori immigrati in Italia è un

Ming, Heidelberg, Winter, 2015, pp. 187-204. Nel convegno del 2016 del DIV, Linda Menninger ha tenuto una conferenza su Mauro Covacich.

17. Gli atti del convegno sono in corso di pubblicazione sulla rivista online Romanische Studien.

18. ADAm, Viktoria, "Nicolai Lilin, Storie sulla pelle", Italienisch: Zeitschrift für italienische Sprache und Literatur, n. 69, 2013, pp. 140-144; Отт, Christine, "Abjekte Fetische. Elena Ferrante Schreiben im Zeichen des vréel”, ibid., n. 75, 2016, pp. 32-59.

19. Mauceri, Maria Cristina, "La letteratura italiana della migrazione nei curricula universitari europei e nordamericani”, in GNISCI, Armando, Moll, Nora (a cura di), Diaspore europee \& Lettere migranti: Primo Festival europeo degli scrittori migranti, Roma, giugno 2002, Roma, Edizioni interculturali, 2002, pp. 145-160, cfr. p. 149. 
fenomeno piuttosto recente. La studiosa Elisabeth Arend ha usato l'espressione "letteratura italofona" a proposito di autori di origine maghrebina in un articolo uscito nel $2009^{20}$. La rivista di cultura italiana contemporanea Zibaldone ha dedicato un numero speciale agli autori italiani di origine africana nel $2011^{21}$. Inoltre, di recente sono stati organizzati due convegni sulla letteratura contemporanea della migrazione e della transculturalità: l'uno da Martha Kleinhans e Richard Schwaderer, in collaborazione con Franca Sinopoli dell'Università Roma La Sapienza, si è svolto nel 2012 a Würzburg; l'altro, organizzato da Rotraud von Kulessa, Nora Moll, Dagmar Reichardt e Franca Sinopoli, si è svolto al Centro italo-tedesco per l'eccellenza europea Villa Vigoni, nell'ottobre 2014²2. I contributi del simposio di Würzburg sono stati pubblicati, in italiano e tedesco, nel 2013, con il titolo Transkulturelle italophone Literatur - letteratura transculturale italofon $a^{23}$, mentre quelli del convegno di Villa Vigoni saranno pubblicati in lingua italiana. Va aggiunto che l'Università di Mannheim ha organizzato una giornata di studi sulla letteratura migrante, transculturale e postcoloniale nell'aprile del 2015, sotto la direzione di Stephanie Neu.

Come si spiega questa attenzione per una letteratura che conta solo poche traduzioni in tedesco e che non è nemmeno canonizzata in Italia? Vari parametri possono contribuire a chiarire questa attenzione per la letteratura transculturale.

Il fenomeno dell'immigrazione in Germania risale agli anni '50 e '60, se non contiamo i flussi migratori successivi alla Seconda Guerra Mondiale, ed è molto probabile che le varie ondate migratorie abbiano creato un certo livello d'interesse, di sensibilità e di accettazione per quanto riguarda la letteratura della migrazione. Negli anni '50, '60 e nei primi anni '70 furono accolti lavoratori

20. ArEnd, Elisabeth, "Eine neue italienische Literatur? Die italophone Literatur", Romanistische Zeitschrift für Literaturgeschichte, n. 33, 2009, pp. 195-238.

21. Italien in Afrika - Afrika in Italien, Zibaldone, n. 52, 2011.

22. Il convegno si intitolava Italien: Gewalt, kulturelles Gedächtnis und Transkulturalität (1990-2014). Il caso italiano: violenza, memoria culturale e transculturalità (1990-2014). Il programma è disponibile sul sito di Villa Vigoni: www.villavigoni.it/events. php?sez_id=12\&pag_id=35\&lang_id=1

23. Kleinhans, Martha, Schwaderer, Richard (a cura di). Transkulturelle italophone Literatur / Letteratura italofona transculturale, Würzburg, Königshausen \& Neumann, 2013. Il volume contiene contributi di studiosi tedeschi, austriaci o che insegnano presso università austriache o tedesche, come Viktoria Adam, Immacolata Amodeo, Gabriella Dondolini, Titus Heydenreich, Maria Kirchmair, Susanne Kleinert, Martha Kleinhans, Rotraud von Kulessa, Dagmar Reichardt, Mario Rossi e Richard Schwaderer, oltre ai contributi di Emma Bond, Daniele Comberiati, Brigitte Le Gouez, Chiara Mengozzi, Nora Moll, Rosanna Morace e Franca Sinopoli. 
provenienti per la maggior parte da Paesi del Mediterraneo come l'Italia, la Spagna, la Grecia e la Turchia. Negli anni '90, arrivarono soprattutto migranti dai Paesi dell'Est, come la Polonia e la Russia, e dai paesi balcanici. Nel 2009, circa 15 degli oltre 80 milioni degli abitanti in Germania erano migranti o figli di migranti, e molti di loro avevano già acquisito la nazionalità tedesca. Fra i residenti stranieri, gli italiani occupavano il quarto posto con una cifra di circa mezzo milione di persone nel $2014^{24}$.

In ambito accademico, gli studi interculturali si sono sviluppati in primo luogo all'interno della germanistica, a partire dagli anni ' $80^{25}$. Molte università tedesche offrono oramai corsi di laurea in comunicazione interculturale. Un filosofo tedesco, Wolfgang Welsch, ha cominciato però a criticare il concetto di interculturalità perché questo - sosteneva - implicava sempre la contrapposizione di due culture e presupponeva l'esistenza di una frontiera stabile tra di esse. Secondo Welsch ${ }^{26}$, in realtà le frontiere tra ambiti culturali tendono a dissolversi perché la cultura globalizzata, alla quale apparteniamo tutti, le mischia. Per tale motivo, un giovane di oggi subisce tante influenze provenienti da varie culture che si sovrappongono.

Già prima del dibattito nato intorno alle posizioni di Welsch, a livello della letteratura stessa sono emersi nuovi fenomeni, come il gruppo di autori di varie nazionalità PoLiKunst, ovvero Associazione Polinazionale di Letteratura e Arte (Polynationaler Literatur und Kunstverein, e.V. 1980-1987) - un'associazione fondata nel 1980 da due autori italiani immigrati in Germania, Carmine Gino Chiellino e Franco Biondi, e dall'autore siro-tedesco Rafik Schami. Nella collana pubblicata da questa associazione uscì anche, in lingua tedesca, la prima raccolta di racconti di Carmine Abate nel $1984^{27}$.

Carmine Gino Chiellino non è solo poeta ma anche studioso della letteratura interculturale in Germania, un concetto che preferisce all'espressione letteratura della migrazione. Chiellino e Immacolata Amodeo, l'attuale direttrice dell'i-

24. Cfr. Destatis, Bevölkerung und Erwerbstätigkeit. Bevölkerung mit Migrationshintergrund. Ergebnisse des Mikrozensus 2014, Wiesbaden, Statistisches Bundesamt, 2015, https:// www.destatis.de/DE/Publikationen/Thematisch/Bevoelkerung/MigrationIntegration/Migrationshintergrund2010220147004.pdf?_blob=publicationFile, p. 7.

25. Cfr. Leskovec, Andrea, Einführung in die interkulturelle Literaturwissenschaft, Darmstadt, Wissenschaftliche Buchgesellschaft, 2011, p. 9. Da più di trent'anni esiste anche un'associazione di germanistica interculturale.

26. Welsch, Wolfgang, "Was ist eigentlich Transkulturalität?", in DarowsKA, Lucyna, Machold, Claudia (a cura di), Hochschule als transkultureller Raum? Beiträge zu Kultur, Bildung und Differenz, Bielefeld, transcript, 2009, pp. 39-66.

27. Авате, Carmine, Den Koffer und weg!, Kiel, Neuer-Malik Verlag, 1984. 
stituzione italo-tedesca Villa Vigoni, hanno promosso gli studi di letteratura interculturale nell'ambito della comparatistica tedesca ${ }^{28}$. Nella società tedesca e negli atenei tedeschi esiste quindi già una certa tradizione di studi interculturali, soprattutto nel campo della comparatistica.

All'interno dei dipartimenti di lingue e letterature romanze, i primi a occuparsi di questo campo sono stati gli studiosi, particolarmente attivi, di letterature francofone.

Per quanto riguarda l'ambito italianistico, nel 1995 è uscita una raccolta di saggi intitolata Letteratura de-centrata. Italienische Autorinnen und Autoren in Deutschland, curata da Caroline Lüderssen e Salvatore A. Sanna e contenente studi sugli autori italiani fuori d'Italia ${ }^{29}$.

A questo campo di studi appartiene anche la tesi di dottorato di ricerca di Loredana Russo Zweiheimische Figuren in der italienischsprachigen Gegenwartsliteratur / Figure biculturali nella letteratura contemporanea in lingua italiana, che analizza, da un lato, autori italiani emigrati in Germania (Carmine Abate e Marisa Fenoglio) ${ }^{30} \mathrm{o}$ germanofoni come il sudtirolese Joseph Zoderer, e dall'altro autori immigrati in Italia come Tahar Lamri, Laila Wadia, o i cosiddetti "postcoloniali”, come Igiaba Scego ed Erminia dell'Oro. Nella sua analisi, Russo si basa sul dibattito tedesco relativo alle teorie dell'interculturalità ed esamina il modo in cui i personaggi biculturali percepiscono e valutano l'elemento della frontiera tra le culture.

28. Chieldino, Carmine Gino, Die Reise hält an, München, Beck, 1988; ID., Literatur und Identität in der Fremde. Zur Literatur italienischer Autoren in der Bundesrepublike, Kiel, Neuer Malik Verlag, 1989; ID., Am Ufer der Fremde. Literatur und Arbeitsmigration 18701991, Stuttgart/Weimar, Metzler Verlag, 1995; ID. (a cura di), Interkulturelle Literatur in Deutschland. Ein Handbuch, Stuttgart/Weimar, Metzler, 2000; ID., Liebe und Interkulturalität. Essays 1988-2000, Tübingen, Stauffenburg Discussion, 2001; ID., Parole erranti. Saggi 1995-2000, Isernia, Cosmo Iannone Edizione, 2001; Amodeo, Immacolata, Die Heimat heißt 'Babylon'. Zur Literatur ausländischer Autoren in der Bundesrepublik Deutschland, Opladen, Westdeutscher Verlag, 1996; EAD., Hörner, Heidrun (a cura di), Zu Hause in der Welt - Topographien einer grenzüberschreitenden Literatur, Sulzbach/Taunus, Ulrike Helmer Verlag, 2010; EAD., Hörner, Heidrun, Kiemle, Christiane (a cura di), Literatur ohne Grenzen. Interkulturelle Gegenwartsliteratur in Deutschland -Porträts und Positionen, Sulzbach/ Taunus, Ulrike Helmer Verlag, 2009.

29. LÜDERSSEn, Caroline, SANNA, Salvatore A. (a cura di), Letteratura de-centrata. Italienische Autorinnen und Autoren in Deutschland. Texte und Analysen, Frankfurt a. M., Diesterweg, 1995.

30. Uno dei romanzi recenti ambientati in Germania che Russo non ha potuto considerare è Ternitti (Milano, Mondadori, 2011) di Mario Desiati. Questo romanzo sulle dure condizioni di vita degli operai italiani emigrati in Germania è stato pubblicato in tedesco dall'editore Wagenbach nel 2012. 
È in corso di pubblicazione la tesi di dottorato di ricerca di Romina Linardi su varie autrici transculturali (Gabriella Kuruvilla, Igiaba Scego, Laila Wadia e Sumaya Abdel Qader) ${ }^{31}$. Altri studi su autori di origine albanese (Maria Zannini) e sul rapporto tra scrittura migrante e percezione pubblica degli autori postcoloniali e transculturali (Stephanie Neu) sono attualmente in corso all'Università di Mannheim. Anche al convegno mondiale dell'ICLA/AILC (International Comparative Literature Association / Association Internationale de Littérature Comparée), tenutosi a Vienna nel 2016, sono stati presentati vari contributi di studiosi tedeschi e austriaci sulla letteratura transculturale in Italia.

Sarebbe auspicabile continuare in futuro lo studio dei fenomeni inter- e transculturali della letteratura italiana, un campo di ricerca che ci sembra molto stimolante in quanto rende manifesto il fatto che la letteratura ha ancora molto da dire sui processi di cambiamento sociale. In questo senso, la letteratura inter- e transculturale può essere facilmente ricollegata al fenomeno più generale di un riorientamento della letteratura italiana verso questioni etiche e di rappresentazioni del reale.

Susanne KLEINERT Università del Saarland, Saarbrücken

31. La pubblicazione della mia allieva Romina Linardi, intitolata Transkulturalität, Identitätskonstruktion und narrative Vermittlung in Migrationstexten der italienischen Gegenwartsliteratur, uscirà probabilmente per i tipi di Peter Lang. Presso la mia cattedra è attualmente in corso una tesi di dottorato di ricerca su Gezim Hajdari. 